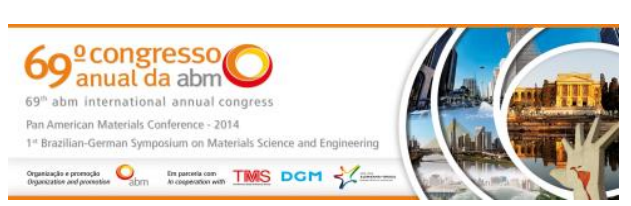

Tema: Materiais cerâmicos, compósitos e poliméricos

\title{
ESTUDO DOS EFEITOS DA RADIAÇÃO GAMA EM EVA POR INFRA VERMELHO E MICROSCOPIA ELETRONICA DE VARREDURA*
}

Elaine Cristina de Azevedo ${ }^{1}$ Viviane Ruffini2

\begin{abstract}
Resumo
Materiais poliméricos sofrem danos estruturais ao serem submetidos à radiação $X$. Estes danos podem causar reticulação, quebra de ligações entre outros danos. Em diversos serviços de radiodiagnostico e de radioterapia é utilizado placa de etileno vinil acetato, EVA, para auxiliar o posicionamento de pacientes crianças ate 2 anos em radiografias de tórax. O objetivo deste trabalho é verificar se este material é adequado para ser utilizado nestes serviços. Para tanto, amostras do material foram submetidas a dose de $25 \mathrm{kGy}$ e avaliados por espectrometria de infra vermelho com transformada de Fourier, e a superfície por medidas de rugosidade e microscopia eletrônica de varredura. Os resultados indicam que o material apresenta degradação com dose de 5 kGy e aumento da rugosidade.
\end{abstract}

Palavras-chave: Radiação gama; Infravermelho e polímeros.

\section{STUDY OF THE EFFECTS OF GAMMA RADIATION ON EVA BY INFRA RED AND SCANNING ELECTRON MICROSCOPY}

\section{Abstract}

Polymeric materials suffer structural damage when subjected to $\mathrm{X}$ radiation. This damage can cause crosslinking, cleavage among other damage. In many diagnostic radiology and radiotherapy service is used ethylene vinyl acetate, EVA board, to assist the positioning of patients during 2 years in children chest radiographs. The objective of this work is to verify that this material is suitable for use in these services. Thus samples of the material were subjected to dose of $25 \mathrm{kGy}$ and evaluated by infrared spectroscopy with Fourier transform, and the surface roughness measurements by electron microscopy and scanning. The results indicate that the material exhibits degradation with $25 \mathrm{kGy}$ dose and increased roughness

Keywords: Gamma radiation; Infrared and Polymer.

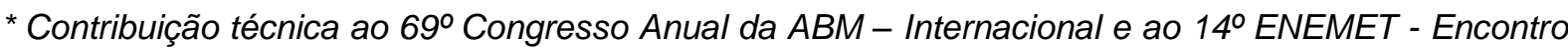
Nacional de Estudantes de Engenharia Metalúrgica, de Materiais e de Minas, 21 a 25 de julho de 2014, São Paulo, SP, Brasil. 


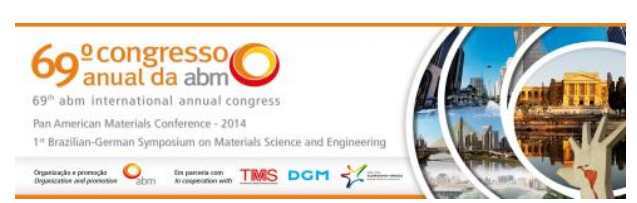

\section{INTRODUÇÃO}

Processos que envolvem a irradiação de polímeros utilizando-se radiação ionizante provocam degradação, que é responsável por alterar características mecânicas e estruturais desses compostos [1-3]. Em salas de radiodiagnóstico e de radioterapia são utilizados diversos tipos de polímeros, sendo necessária a avaliação dos danos causados pela longa exposição dos mesmos [4].

Embora na maioria dos setores de radiodiagnóstico sejam utilizados os raios $X$, utilizou-se nesse estudo a radiação gama, pois os efeitos de ambas são os mesmos em polímeros [4]. Cada exposição em exames radiológicos executada pela radiação $X$, em salas de exames, possui baixa dose, mas provoca efeitos cumulativos ao longo do tempo [5].

A interação da radiação ionizante com polímeros resulta na formação de moléculas ionizadas e moléculas excitadas que posteriormente se recombinam ou dissociam para produzir radicais livres ou íons [6]. Gerando reações químicas que podem provocar modificações permanentes na estrutura do material irradiado [7].

Entre os efeitos causados pela radiação quando esta incide em polímeros, ocorre a reticulação, na qual o polímero tem seus átomos arrancados de sua posição na estrutura causando a criação de pontos ativos que podem gerar ramificações, e a degradação, a qual a estrutura pode ser degradada, ocasionando perda de resistência mecânica, ou, ainda, podem ter a estrutura distorcida em virtude da formação de vazios [8]. Ainda que a reticulação e a degradação ocorram simultaneamente durante a irradiação do polímero, um destes processos é predominante, dependendo da estrutura química do polímero, da dose de radiação e das condições nas quais o material é irradiado [7]. A espectroscopia na região do infravermelho pode fornecer informação importante nas mudanças de ligações químicas e a medida da rugosidade de uma superfície que fica em contato com pacientes é importante tanto para a assepsia como para o conforto.

O etileno vinil acetato, EVA, surgiu nos Estados Unidos na década de 1950 e teve seu uso expandido na década de 70 no ramo dos calçados, sendo uma alternativa ao uso do couro, está presente em solados, palmilhas, brinquedos, material escolar, materiais médicos entre outros [9].

O objetivo desse trabalho é avaliar o efeito da radiação em EVA, que está presente em diversos equipamentos da sala de radiodiagnóstico [10]. Para avaliar se ocorreu degradação após a irradiação, amostras foram irradiadas com dose de 25 kGY, dose de esterilização, as ligações químicas foram analisadas através da espectroscopia de infravermelho com transformada de Fourier e avaliado alterações na superfície através da medida da rugosidade e micrografia de microscopia eletrônica de varredura.

\section{MATERIAIS E MÉTODOS}

\subsection{EVA}

Obtida no comercio local, foram cortadas e embaladas para serem submetidas a radiação gama.

\footnotetext{
* Contribuição técnica ao $69^{\circ}$ Congresso Anual da ABM - Internacional e ao 14ํㅡㄹ ENEMET - Encontro Nacional de Estudantes de Engenharia Metalúrgica, de Materiais e de Minas, 21 a 25 de julho de 2014, São Paulo, SP, Brasil.
} 


\subsection{Radiação Gama}

A irradiação gama em doses de 5 kGy, 10 kGy, 15 kGy, 20 kGy e 25 kGy foi aplicada pela empresa Embrarad - Cotia-SP, com uma fonte industrial de Cobalto 60 MDS Nordion's JS-9600.

\subsection{Espectroscopia de infravermelho}

Foi utilizado o espectrômetro de infravermelho Nicole, Modelo 5SXC-FTIR, com resolução de $4 \mathrm{~cm}^{-1}$.

\subsection{Microscopia Eletrônica de Varredura}

A Microscopia eletrônica de varredura foi realizada nas amostras de EVA com o equipamento ZEISS modelo EVO MA 15.

\subsection{Rugosidade das amostras}

A medida de rugosidade das amostras foi feita pelo equipamento Talysurf CCI Lite Non-contact 3D Profiler - TAYLOR HOBSON.

\section{RESULTADOS E DISCUSSÃO}

Na Figura 1 é mostrado o espectro obtido por ATR da amostra de EVA sem irradiar, sobreposto ao amostra irradiada com $25 \mathrm{kGy}$, pode se observar a banda característica de grupos hidrocarboneto na região de $2900 \mathrm{~cm}^{-1}$, na região de $1300 \mathrm{~cm}^{-1}$, grupos carbonila na região de $1600 \mathrm{~cm}^{-1}$ e grupos hidrocarbonetos ligados ao oxigênio da região de $1200 \mathrm{~cm}^{-1}$. A presença de banda de absorção na região de $3300 \mathrm{~cm}-1$ está relacionada ao oxigênio presente na atmosfera e as demais bandas estão relacionadas aos plastificantes e aos aditivos presentes na estrutura do EVA.

Observa se nos espectros da Figura 1, que todos os picos que aparecem na amostra não irradiada estão presentes também nos espectros da amostra irradiada, diferindo apenas nas intensidades. É possível observar que a intensidade de absorção da radiação em cada pico aumenta com o aumento da dose. Esse fato indica que as ligações existentes nesse polímero absorvem parte da radiação incidente, diminuindo a transmitância do feixe para o detector. Essa radiação absorvida pelo polímero provoca o enfraquecimento das ligações desencadeando a degradação do material.

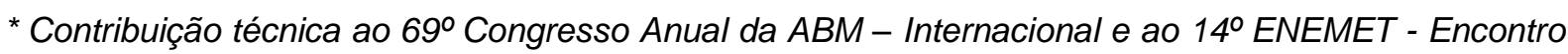
Nacional de Estudantes de Engenharia Metalúrgica, de Materiais e de Minas, 21 a 25 de julho de 2014, São Paulo, SP, Brasil.
} 

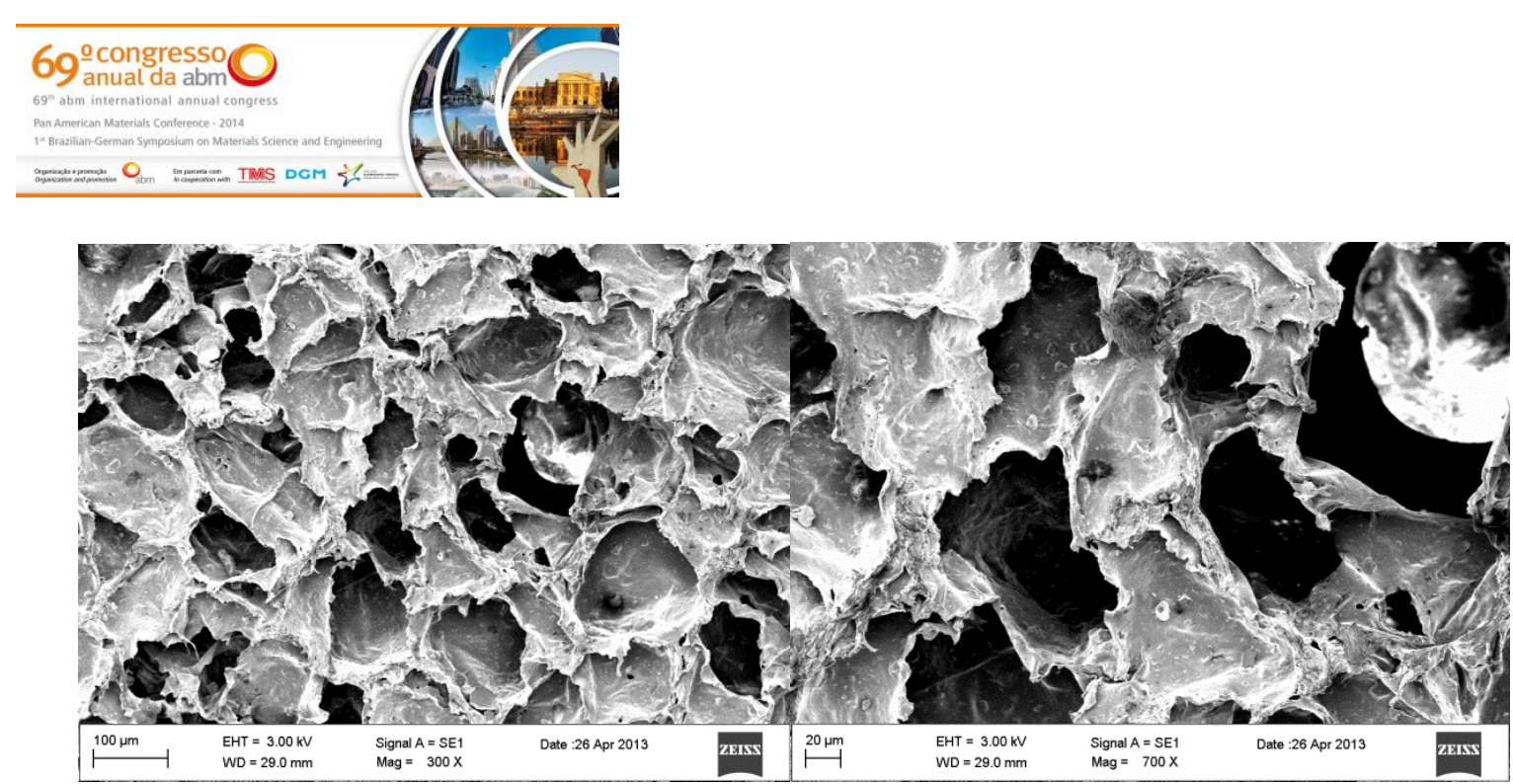

(a)

(b)

Figura 3 - Micrografia de microscopia eletrônica de varredura da superfície do EVA irradiada com 25 kG (a) $100 \mathrm{m \mu}$ e (b) $20 \mathrm{m \mu}$.

A Tabela 3 apresenta os valores de rugosidade média encontrado para as amostras de EVA.

Tabela 3 - Rugosidade superficial Média (Sa) das amostras de EVA

\begin{tabular}{|c|c|}
\hline Dose recebida (kGy) & $\mathbf{S a}\left(\boldsymbol{\mu m}^{2}\right)$ \\
\hline Não irradiada & 8.275 \\
\hline 25 & 20.99 \\
\hline
\end{tabular}

Os valores encontrados para a rugosidade superficial da amostra irradiada é superior ao da amostra não irradiada, o que indica aumento das irregularidades existentes na superfície do polímero após a irradiação, evidenciando a ocorrência da degradação no material. Para maior proteção dos pacientes, materiais que tenham sua estrutura degradada com a radiação não devem ser usados em setores de Radiologia Médica.

As Figuras 4a e 4b apresentam as imagens obtidas das medições de rugosidade superficial, onde é possível observar o aumento da irregularidade na superfície do polímero após a irradiação.

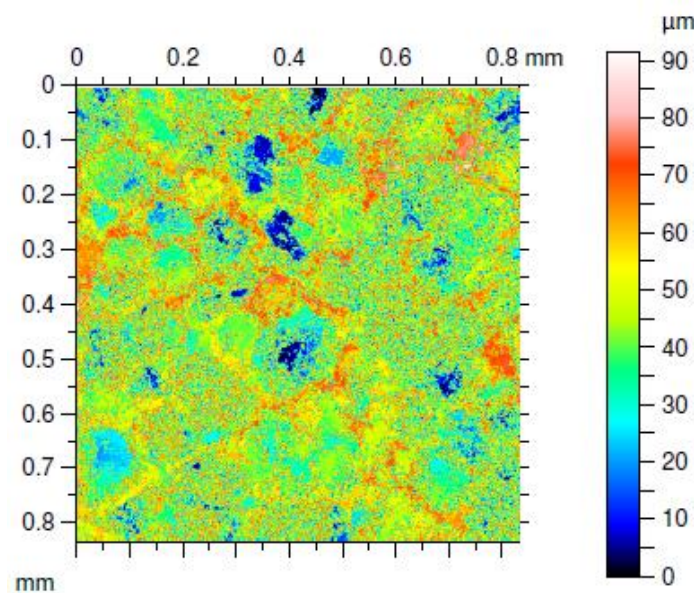

(a)

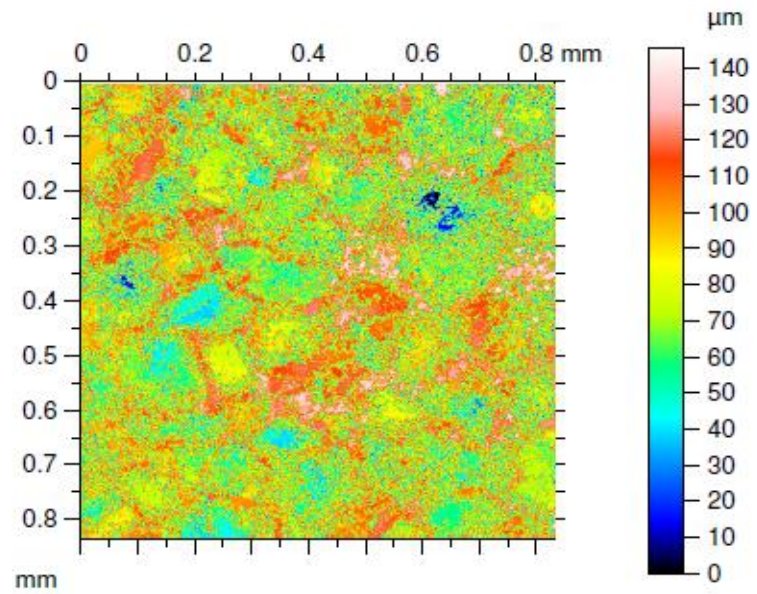

(b)

Figura 4 - Imagem da rugosidade superficial (a) sem irradiar (b) irradiada com dose de 25kG.

\footnotetext{
* Contribuição técnica ao 69 Congresso Anual da ABM - Internacional e ao 14ํㅡㄹ ENEMET - Encontro Nacional de Estudantes de Engenharia Metalúrgica, de Materiais e de Minas, 21 a 25 de julho de 2014, São Paulo, SP, Brasil.
} 


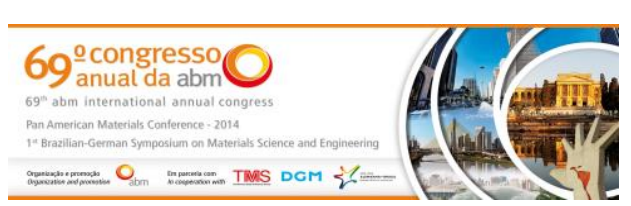

O aumento da coloração amarela e, principalmente, da coloração vermelha nas amostras após a irradiação com radiação gama indica que houve degradação do material.

\section{CONCLUSÃO}

O EVA é degradado pela radiação não sendo indicado que seja utilizado em acessórios de posicionamento usados em serviços de radiodiagnósticos e de radioterapia pois além da degradação apresentam pontos de acumulo de sujidade o que torna o processo de esterilização ineficaz.

\section{Agradecimentos}

Gostaríamos de agradecer ao CNPq, CAPES e Fundação Araucária pelo auxilio financeiro aos laboratórios envolvidos.

\section{REFERÊNCIAS}

1 Makuuchi K., Radiation Processing of Polymer Materials and its Industrial Applications. John Wiley \& Sons, 2011.

2 ASTM Committee D-2 on Petroleum Products and Lubricants, The Effects of Polymer Degradation on Flow Properties of Fluids and Lubricants Containing Polymers: A Symposium on Petroleum Products and Lubricants, Atlantic City, N.J., Jan. 26-31, 1964. ASTM, 1965.

3 Kausch H. Radiation Effects on Polymers for Biological Use. Series: Advances in Polymer Science, Vol. 162. Springer-Verlag Berlim Heidelberg, 2003.

4 Goulin E, Rosa M, Azevedo E. Análise de polietileno irradiado com radiação gama por FTIR. In: 20ํㅡㄹ CBECIMAT, 2012, Joinville. 20 CBECIMAT, 2012.

5 Charlesby A. Atomic Radiation and Polymer. Editora Pergamom, London, 1960.

6 Colombo MAS. Estudo do efeito da Radiação lonizante sobre as propriedades mecânicas da Poliamida 6,6. Tese de mestrado em Ciências na Área de Tecnologia Nuclear. Instituto de Pesquisas Energéticas e Nucleares. São Paulo, 2006.

7 Silva LGA. Energia Nuclear - Polímeros. Instituto de Pesquisas Energéticas e Nucleares e Comissão Nacional de Energia Nuclear. Centro de Tecnologia das Radiações. São Paulo, 2010.

8 Azevedo EC. Efeito da Radiação nas Propriedades Mecânicas do Adesivo de Poliuretana Derivado do Óleo de Mamona. Tese de doutorado na área de Ciência e Tecnologia dos Materiais. PIPE, Setor de Tecnologia da Universidade Federal do Paraná, 2009.

9 Eureka Indústria e Comércio Ltda., EVA. São Paulo, 2009. Disponível em: http://www.eurekaeva.com.br/sobre-eva.html. Acesso em: 04/03/2014.

10 Light KL, Halperin EC. Pediatric Radiation Oncology. Lippincott Williams \& Wilkins, 5a Ed, 2010.

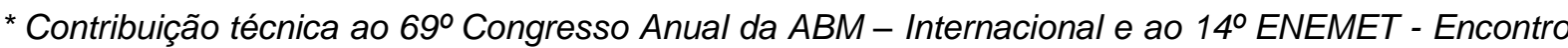
Nacional de Estudantes de Engenharia Metalúrgica, de Materiais e de Minas, 21 a 25 de julho de 2014, São Paulo, SP, Brasil.
} 